\title{
Kräfte und Massnahmen bündeln für mehr Patientensicherheit
}

\author{
Michael Wehrlia , Thierry Fumeaux ${ }^{b}$, Hans Ulrich Rothen ${ }^{c}$, Martin Balmer ${ }^{d}$ \\ ${ }^{a}$ Geschäftsführender Präsident SGI; ${ }^{b}$ Prof. Dr. med., Präsident Ärzte SGI, Mitglied FMH; ${ }^{c}$ Prof. Dr. med., Vizepräsident Kommission Datensatz SGI, \\ Lead Qualitätskommission SGI, Mitglied FMH; ${ }^{d}$ Beisitzer Vorstand SGI, Präsident Qualitätskommission SGI
}

Die Schweizerische Gesellschaft für Intensivmedizin (SGI) hat es sich schon früh zu einer ihrer Hauptaufgaben gemacht, die Behandlungsqualität und die Patientensicherheit auf Intensivstationen in der Schweiz zu sichern und zu verbessern. Bereits 1976 begann die Fachgesellschaft damit, landesweit Intensivstationen nach bestimmten Richtlinien anzuerkennen. Das interprofessionelle Zertifizierungsverfahren von Intensivstationen, das mit den Jahren aus diesem Anerkennungsprozess entwickelt wurde, ist bis heute weltweit einmalig.

Eng mit der Zertifizierung von Intensivstationen verknüpft ist der Minimale Datensatz der SGI (MDSi). Der MDSi definiert und erfasst bestimmte Kennzahlen einer ICU wie etwa die Überlebensrate kritisch kranker Patienten und Patientinnen nach dem Aufenthalt auf einer bestimmten Intensivstation. Durch die anonymisierten Daten können Intensivstationen schweizweit miteinander verglichen werden. Anhand des Datensatzes kann aber auch eingeschätzt werden, ob eine

\section{Durch die anonymisierten Daten können Intensivstationen schweizweit miteinander verglichen werden.}

bestimmte Intensivstation von der Zertifizierungskommission der SGI anerkannt wird oder nicht. Die Kommission Datensatz, die für den MDSi zuständig ist, ist eine von fünf Kommissionen, die sich heute im Ressort "Qualität» der SGI um das Thema Patientensicherheit kümmern.

\section{Den eigenen Horizont erweitern}

Trotz dieser langjährigen Tradition und Bedeutung der Patientensicherheit in der Fachgesellschaft lohnt es sich, über den eigenen Tellerrand zu schauen und Expertinnen und Experten aus anderen Disziplinen und Branchen zu konsultieren, um mit Qualitätssicherung und -entwicklung die Patientensicherheit weiter zu verbessern. Aus diesem Grund fand das diesjährige SGI Symposium am 27. April als Plattform für interprofessionellen und interdisziplinären Austausch zum
Thema «Patientensicherheit» statt. Unterstützung erhielt die SGI in diesem Jahr von der FMH und der Stiftung für Patientensicherheit, die freundlicherweise zusammen das Patronat für das Symposium übernommen haben.

Die Analyse von Fehlern ist für Qualitätsverbesserung ein gängiger Ausgangspunkt. Wie Bernhard Frey vom Universitätskinderspital Zürich zum Thema Medikation erwähnt, können Behandlungsfehler schon alleine dadurch vermieden werden, dass man nicht zu viele Medikamente verschreibt. Erst im letzten Jahr hat die SGI im Rahmen der Initiative «Smarter Medicine» eine Liste mit neun Massnahmen publiziert, deren Verordnung auf den Intensivstationen in der Schweiz grundsätzlich überdacht werden soll, weil für den Patienten oder die Patientin unter Umständen kein Nutzen besteht. Mehrere dieser Massnahmen beziehen sich auf die Verordnung von Medikamenten. Eine klare, unmissverständliche Etikettierung reduziert Verschreibungsfehler zusätzlich: Ein Medikament sollte äusserlich nicht aussehen wie ein anderes, die angegebene Dosierung sollte klar und verständlich sein. Elektronische Patientendatenmanagementsysteme (PDMS) würden die Fehlerquote weiter senken, so Bernhard Frey. Doch sind diese noch längst nicht in allen Spitälern im Einsatz. Bis dies der Fall ist, kann der Miteinbezug einer weiteren Kontrollinstanz dazu beitragen, dass Fehler vermieden werden. Im Universitätskinderspital Zürich begleitet deshalb häufig ein Spitalapotheker die Visite.

Dass die Involvierung aller beteiligten Berufsgruppen und Disziplinen zu einer verbesserten Sicherheitskul- 
tur führt, bestätigen auch Alison Lovatt und Ali Cracknell, die in Nordengland sogenannte Huddles eingeführt haben. An diesen Kurztreffen, die täglich zur gleichen Zeit am selben Ort und vorzugsweise im Stehen durchgeführt werden, sollen in fünf bis fünfzehn Minuten Risikopatienten und -patientinnen besprochen werden. Dadurch, dass das gesamte interprofessionelle Team anwesend ist, erhöht sich die Patientensicherheit zusätzlich. Das bestätigt auch Michael Simon von der Universität Basel in seinem einführenden Übersichtsreferat. Huddles, so Alison Lovatt und Ali Cracknell, sind dazu in der Lage, unerwünschte Ereignisse auf effiziente Weise zu verhindern, die Kommunikation in der Belegschaft und am Ende die Patientensicherheit zu verbessern.

\section{Zusammen für mehr Patientensicherheit}

Auch in der Qualitätsarbeit sind Kooperationen verschiedener Organisationen und Disziplinen durchaus sinnvoll. Die Schweizerische Akademie für Qualität in der Medizin (SAQM) hat 2016 eine Qualitätscharta veröffentlicht, die seitdem von 70 Organisationen - unter anderem von der SGI - unterzeichnet wurde. Dieses Versprechen zur Qualität beruht, wie Christoph Bosshard, Vizepräsident der FMH, erwähnt, auf den drei Pfeilern Transparenz, Verbindlichkeit und Nachhaltigkeit. Unterzeichnende Gruppierungen garantieren, bestehende und neue ärztliche Aktivitäten zur Förderung der Qualität in der Medizin sowie die Qualität der ärztlichen Leistungserbringung sichtbar zu machen. Im Zeichen der Verbindlichkeit entwickeln sie zudem eine Qualitätsstrategie und evaluieren die Arbeiten regelmässig in einem Qualitätsbericht. Die SGI ist hier mit der Gründung ihrer interprofessionellen Qualitätskommission im letzten Herbst, die als erste Aktion eine eigene Qualitätscharta präsentiert hat, sogar einen Schritt weitergegangen.

Zudem versichern Organisationen mit ihrer Willensbekundung, die Qualitätsstrategie zu einem längerfristigen Projekt zu erklären, das die Qualitätsentwicklung sicherstellt. Ausserdem ist die Qualitätsarbeit für jene Organisationen, welche die Charta unterschrieben haben, ein fester Bestandteil der ärztlichen Aus-, Weiter- und Fortbildung.

\section{Aus Daten lernen}

Laut Bernard Burnand von der Universität Lausanne ist es wichtig, unerwünschte Ereignisse zu erfassen. Doch ist es dabei von grösster Bedeutung, dass die Dokumentation einfach realisierbar ist. Digitale Werkzeuge können dabei behilflich sein. Im Bereich der Spi- talinfekte, so Jonas Marschall vom Inselspital in Bern, bietet Swissnoso als Nationales Zentrum für Infektprävention etwa verschiedene Anwendungen oder Module an, mit denen nosokomiale Infektionen praxis-

\section{Dadurch, dass das gesamte interprofessionelle} Team anwesend ist, erhöht sich die Patientensicherheit zusätzlich.

tauglich und kostengünstig überwacht und verhindert werden können, um so am Ende die Patientensicherheit weiter zu steigern.

\section{Strukturelle Veränderungen statt indivi- dueller Massnahmen}

In seinem Referat über die Steigerung von Patientensicherheit durch die Verbesserung von Design erwähnt David Schwappach von der Stiftung für Patientensicherheit, dass häufig weniger aufwendige, individuumsbezogene Interventionen im Zentrum stehen, wo eigentlich tiefer greifende, strukturelle Veränderungen nötig wären, um tatsächlich Fortschritte in der Patientensicherheit zu bewirken. So seien Schulungen, SOPs und Merkblätter zwar einfach ausgeführt oder erarbeitet, in vielen Fällen jedoch praktisch erfolglos. Als Illustration dient David Schwappach, dessen Referat auf der SGI-Website als Webcasting zugänglich ist, die Vorbereitung von Anästhetika vor Operationen [1]. Um Medikationsfehler in der Anästhesie zu bekämpfen, haben Ärzte in Zusammenarbeit mit Industriedesignern ein Spritzentablett entwickelt, das mit Hilfe von markierten Vertiefungen das Verrutschen von Spritzen verhindert und mit einer Farbführung die Zuordnung der einzelnen Medikamente sichert. Auf diese

\section{Um Medikationsfehler in der Anästhesie zu} bekämpfen, haben Ärzte in Zusammenarbeit mit Industriedesignern ein Spritzentablett entwickelt.

Weise werden Ärztinnen, Ärzte und Pflegefachpersonen entlastet, sie können sich stärker auf den Patienten oder die Patientin konzentrieren, die Sicherheit wird erhöht.

Auch für Anthony Staines von der Fédération des Hôpitaux Vaudois (FHV) greifen individuelle Massnahmen zu kurz. Wer die Sicherheitskultur auf einer Station nachhaltig verbessern möchte, sollte sich eines multimodalen Ansatzes bedienen, der viele einzelne Mittel mit demselben Ziel vereint. So konnte die Handhygiene an verschiedenen Spitälern im Kanton Waadt 
etwa dadurch gesteigert werden, dass einerseits mit Broschüren, Postern und Aufklebern auf eine gründliche und regelmässige Desinfektion hingewiesen

\section{Anstatt nur Fehler zu analysieren und so zu einer erhöhten Patientensicherheit beizutragen, sollten auch die Erfolge untersucht werden.}

wurde, andererseits aber auch die Anzahl der Dosierspender auf den Stationen erhöht wurde.

\section{Kulturwandel}

Sven Ständer vom Spital Männedorf appelliert für einen grundsätzlichen Kulturwandel. Anstatt nur Fehler zu analysieren und so zu einer erhöhten Patientensicherheit beizutragen, sollten auch die Erfolge untersucht werden. So wird nicht vermieden, dass etwas misslingt, sondern gewährleistet, dass alles korrekt vonstattengeht. In diesem Konzept namens «Safety 2.0» wird reaktives Qualitätsmanagement zu einer proaktiven Angelegenheit. Angestellte werden zu Ressourcen, die dazu genutzt werden, den alltäglichen Ablauf zu analysieren und das seltene Versagen zu verstehen.
In der Schlussrunde des Symposiums sind sich alle Teilnehmenden der Komplexität nachhaltig wirkender Massnahmen bewusst. Die Verbesserung der Patientensicherheit bleibt für alle involvierten Berufsgruppen eine grosse Herausforderung - systemische teambezogene Analysen und Massnahmen sollen die Sicherheitskultur in den schweizerischen Intensivstationen auch in Zukunft vertiefen - die Qualitätskommission der SGI arbeitet hierzu an der Umsetzung ihrer Strategie.

\section{Literatur}

1 Grigg EB, Martin LD, Ross FJ, et al. Assessing the Impact of the Anesthesia Medication Template on Medication Errors During Anesthesia: A Prospective Study. Anesthesia Analgesia. 2017;124:1617-25; doi: 10.1213/ANE.0000000000001823.

\section{Jahrestagung der SGI}

Vom 19. bis 21. September findet in Interlaken die SGI Jahrestagung mit interessanten interprofessionellen Workshops und Referaten statt. Wir würden uns freuen, einige von Ihnen in Interlaken begrüssen zu dürfen. Weitere Informationen zum Kongress und zur SGI finden Sie unter www.sgi-ssmi.ch 